\title{
Alpha wave of sleep electroencephalogram analysis based on multiscale sign series entropy
}

\author{
Jianhui Jiang ${ }^{1}$ Shitong Wang ${ }^{1} \quad$ Fengzhen $\mathrm{Hou}^{2, a} \quad \mathrm{Jin}^{2} \mathrm{i}^{3} \quad$ Jun \\ Wang $^{1, b}$
}

1 Image Processing and Image Communications Key Lab., Nanjing Univ. of Posts \& Telecomm., Nanjing 210003, China

2 School of Science, China Pharmaceutical University, Nanjing 210009, China

3 College of Physics and Information Technology, Shaanxi Normal University, Xian 710062, China

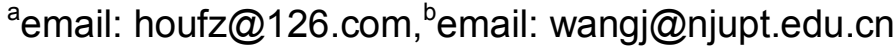

Key words: Sleep Electroencephalogram, multiscale sign series entropy, clinical diagnosis.

\section{Abstract}

Sleep Electroencephalogram (Sleep EEG) detection and treatment can provide the basis for clinical diagnosis and treatment. According to the non-stationary random character of EEG itself, the paper proposed multiscale sign series entropy (MSSE) method and applied it to the state of sleep EEG analysis. Numerical results showed that, MSSE method can effectively differentiate awake period $\alpha$ wave and sleep stage awave even if under the influence of the noise. The results show that the algorithm can aid in clinical diagnosis of sleep EEG.

\section{Introduction}

Under the status of sleeping, the brain is basically in the spontaneous activity state because of shielding most of the interference. At this time brain waves are similar to the awake stage, it indicates that the brain is in active state. So it provides a good platform for brain, cognition, brain function and structure of brain ${ }^{[1]}$. In the past decades, sleep studies mainly focus on the factors of how to affect sleep and how to affect other physiological and cognitive functions by sleeping ${ }^{[2-4]}$.

Combined with multiscale ${ }^{[5]}$ and the symbol sequence entropy concept (sign series entropy, SSE) ${ }^{[6]}$, multiscale sign series entropy was proposed. The algorithm was used to analyze sleep alpha component and awake alpha component of the EEG. We study the influence to MSSE value of alpha wave on awake and sleep from the viewpoints of the data length change, word length $\mathrm{m}$ change, noise effects. Sleep EEG is discussed and the numerical results show that this method can effectively detect the change of short-term $\alpha$-wave in awake and sleep period.

\section{Method of multiscale sign series entropy}

Potential changes of EEG signals are non-stationary random. We represent potential change variation in three ways. There are three symbols representing the EEG changes direction.

$$
\mathrm{x}(\mathrm{i})= \begin{cases}0, & \mathrm{R}(\mathrm{i}+1)<\mathrm{R}(\mathrm{i}), \\ 1, & \mathrm{R}(\mathrm{i}+1)=\mathrm{R}(\mathrm{i}), \\ 2, & \mathrm{R}(\mathrm{i}+1)>\mathrm{R}(\mathrm{i}),\end{cases}
$$


$\mathrm{x}(\mathrm{i})=0$ indicates the decline of a potential; $\mathrm{x}(\mathrm{i})=1$ indicates the invariant of a potential; $\mathrm{x}$ (i) $=2$ indicates the rise of potential. Three symbols represent only three states in symbol sequence generation and numerical size does not make sense. Through symbolizing of the potential changes, it only retains the change of direction information. For revealing the law of timing and structure, we used the sliding window method to construct vector sequence for symbolic direction signal ( $\mathrm{m}$ word width):

$X(i)=[x(i), x(i+1), \ldots \ldots x(i+(m-1))], i=1,2,3 \ldots \ldots N-m$.

Vector X(i) represents the continuous change of direction. Continuous variable common species has $\mathrm{M}=3^{\mathrm{m}}$ possible modes with $\mathrm{m}$ word width, the statistical probability of each mode appears:

$$
\mathrm{p}_{\mathrm{j}}=\frac{\mathrm{N}_{\mathrm{j}}}{\mathrm{N}-\mathrm{m}}, \mathrm{j}=1,2,3 \quad \ldots \ldots \mathrm{M}
$$

When $\mathrm{m}=2$, it will appear a total of nine kinds of modes as Figure 1. Each changes mode has a probability and the model probability is shown in Figure 1.

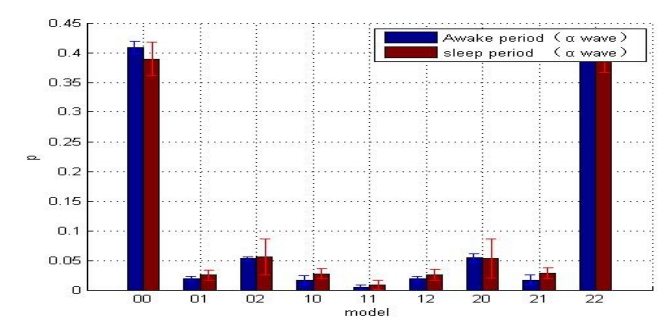

Figure 1: Statistical distribution of modes for all samples

Where $\mathrm{N}_{\mathrm{j}}$ is the number of the $\mathrm{j}$-th modes, entropy is calculated as [18]:

$$
\operatorname{SSE}(m)=-\sum_{j=1}^{M} p_{j} \log { }_{2} p_{j} .
$$

Multiscale method for processing EEG time series is shown as follows: given a time series $\{\mathrm{x}$ $(1), \ldots, x(i), \ldots, x(N)\}$, building a continuous sequence determined by the scale factor $t$, the following equation is:

$$
\mathrm{y}^{(\mathrm{t})}(\mathrm{j})=1 / \mathrm{t} \sum_{\mathrm{i}=(\mathrm{j}-1) \mathrm{t}+1}^{\mathrm{jt}} \mathrm{x}(\mathrm{i}), \quad 1 \leq \mathrm{j} \leq \mathrm{N} / \mathrm{t} \text {. }
$$

The scale factor $t=1$ sequence $y(j)$ is simplified as the original time series $x(i)$. Each length scale of the time series is equal to the original sequence length divided the scale factor. After multiscale algorithm and using SSE, we can acquired the MSSE results of EEG. 


\section{Numerical Analysis}

Using collected EEG to validate the algorithm, Figure 2 shows SSE results of four groups of awake $\alpha$ component and four groups of sleep stage I alpha component while six consecutive segment data length is 500 points. The horizontal axis is the sample number of Group 1 4 alpha component and group 1 4 alpha component during awake and sleep stage I. The vertical axis represents SSE results of each data segment. It can be seen that the result of the same object is very close to the adjacent data segments, which shows that the method can get a effective characteristic parameter from the shorter data section.

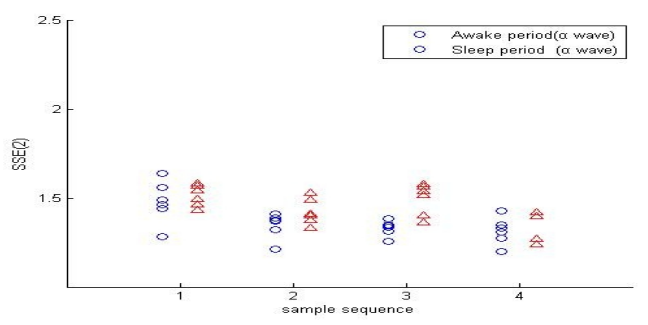

Figure 2: Comparison between the adjacent continuous short data segments.

In Figure 3(a), we used the mean and standard deviation respectively to describe results of 4 samples of 500 points during awake alpha component and sleep stage 1 alpha component. It shows that SSEA has the effectiveness on brain electrical signal and has a certain clinical practicability. In Figure 4(b), it shows the influence of word length width $m$ to the results of SSEA. The data length is 500. Under the condition of data length, if increasing the length width, pattern $\left(\mathrm{M}=3^{\mathrm{m}}\right)$ will increase and it will lead to increase of SSEA values. But in the analysis of the $m=2 \sim 6$ range, from Figure 3(b) we showed that the SSEA can effectively distinguish the awake alpha component and sleep alpha component.

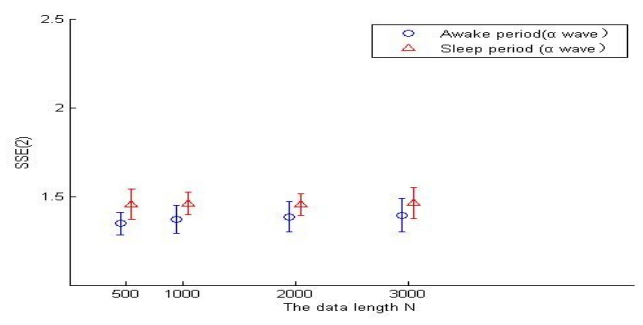

Figure 3(a): The impact of the data length $\mathrm{N}$ on SSEA

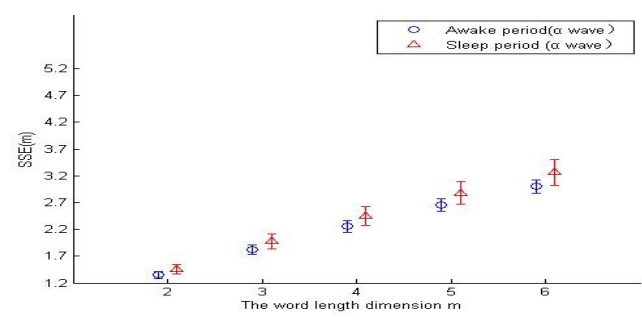

Figure 3(b): The effects of word length dimension m on SSEA

The original data is disposed using the multiscale processing for the confirmed length sequence, the signal sequence is coarse-grained according to the scale factor t. Then we analyze 
each coarse-grained EEG signals with SSE algorithm and the results are shown in figure 4. It can be shown that MSSE can distinguish between the awake alpha component and sleep EEG alpha component. Figure 4 shows that, as the scale increasing, the symbol sequence entropy synchronous decrease for awake and sleep EEG, but the entropy values of sleep stage are always higher than the wake stage.

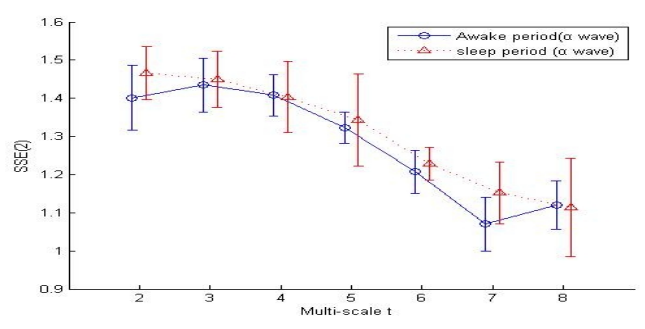

Figure 4: Analysis of the SSEA algorithm after the treatment of multiscale

\section{Conclusions}

We propose multiscale sign series entropy and apply it to sleep EEG analysis. The paper studies the SSEA value change regulation according to the data length, word length and dimension changes. Meanwhile, it studies the influence of random noise to SSEA values on sleep EEG. The numerical calculation results show that SSEA is robust. It illustrates that our proposed MSSE can distinguish the alpha component of sleep and awake stage alpha component of the EEG signals, which can assist the research of sleep staging.

\section{Acknowledgements}

In this paper, the Project supported by the National Natural Science Foundation of China (Grant Nos. 61271082,61201029,61102094,61401518), the Natural Science Foundation of Jiangsu Province (Grant Nos.BK20141432) , the Foundation of Nanjing General Hospital of Nanjing Military Command (2014019) and the Fundamental Research Funds for the Central Universities (FY2014LX0039).

\section{References}

[1] Siegel, J. M., Clues to the functions of mammalian sleep. Nature, 437(7063),pp. 1264-1271, 2005.

[2] Czeisler, C. A., Duffy, J. F., Shanahan, T. L., Brown, E. N., Mitchell, J. F., Rimmer, D. W., Ronda, J. M., Silva, E. J., Allan, J. S., Emens, J. S., Dijk, D. J. \& Kronauer, R. E., Stability, precision, and near-24-hour period of the human circadian pacemaker. Science, 284(5423),pp. 2177-2181, 1999.

[3] Lo, C. C., Amaral, L. A. N., Havlin, S., Ivanov, P. C., Penzel, T., Peter, J. H. \& Stanley, H. E., Dynamics of sleep-wake transitions during sleep. Europhysics Letters, 57(5),pp. 625-631, 2002.

[4] Huber, R., Ghilardi, M. F., Massimini, M. \& Tononi, G., Local sleep and learning. Nature, 430(6995),pp. 78-81, 2004.

[5] Costa, M., Goldberger, A. L. \& Peng, C. K., Multiscale entropy analysis of complex physiologic time series. Physical Review Letters, 89(6),pp. 068102, 2002. 
[6] Bian, C., Ma, Q., Si, J., Wu, X., Shao, J., Ning, X. \& Wang, D., Sign series entropy analysis of short-term heart rate variability. Chinese Science Bulletin, 54(24),pp. 4610-4615, 2009. 